\title{
Metabolic defects in splenic B cell compartments from patients with liver cirrhosis
}

\author{
Man Huang ${ }^{1,2,3}$, Xiaoju Liu ${ }^{4,5}$, Haocheng Ye ${ }^{1}$, Xin Zhao ${ }^{2}$, Juanjuan Zhao ${ }^{1}$, Yang Liu', Xiaomeng He ${ }^{6}$, Mengmeng Qu ${ }^{6}$,

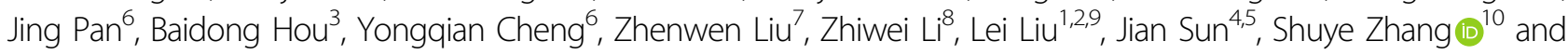 \\ Zheng Zhang (1) $^{1,2,9,11}$
}

\begin{abstract}
Liver cirrhosis is associated with defective vaccine responses and increased infections. Dysregulated B cell compartments in cirrhotic patients have been noticed but not well characterized, especially in the spleen. Here, we comprehensively investigated B cell perturbations from the spleens and peripheral blood of cirrhotic patients. We found that liver cirrhosis significantly depleted both switched and nonswitched splenic memory B cells, which was further confirmed histologically. Bulk RNA-seq revealed significant metabolic defects as the potential mechanism for the impaired splenic B cell functions. Functionally, the splenic memory B cells from cirrhotic patients showed strong metabolic defects and reduced proliferation compared with those from healthy controls. Thus, liver cirrhosis extensively disturbs the splenic and peripheral B cell compartments, which may contribute to defective humoral immunity during liver cirrhosis.
\end{abstract}

\section{Introduction}

The human liver plays important roles in maintaining immune homeostasis ${ }^{1}$. During liver cirrhosis (LC), the immune functions of the liver are largely compromised ${ }^{2}$. Cirrhosis-associated immune dysfunction (CAID) underlies many pathological events, leading to severe complications and mortality in cirrhotic patients ${ }^{3}$. For example, in patients with advanced liver cirrhosis, immune deficiency generally contributes to increased susceptibility to microbial infections, frequently leading to patient death ${ }^{4}$. Alternatively, patients with compensated liver cirrhosis can suffer from acute-on-chronic liver failure (ACLF), which is initiated by various triggering events ${ }^{5}$. The onset

\footnotetext{
Correspondence: Lei Liu (liulei3322@aliyun.com) or Jian Sun (sunjian@smu.edu.cn) or Shuye Zhang (zhangshuye@shphc.org.cn)

'Institute for Hepatology, National Clinical Research Center for Infectious Disease,

Shenzhen Third People's Hospital, 518112 Shenzhen, Guangdong, China

${ }^{2}$ The Second Affliated Hospital, School of Medicine, South University of Science and Technology, 518100 Shenzhen, China

Full list of author information is available at the end of the article

These authors contributed equally: Man Huang, Xiaoju Liu, Haocheng Ye,

Xin Zhao

Edited by Y. Shi
}

of ACLF is related to CAID, during which imbalanced immunity perplexingly causes both hyperinflammation and immune paralysis, leading to dysregulated inflammatory injury during the triggering phase and failed immune defenses during the regenerative phase ${ }^{5,6}$. In addition, cirrhotic patients respond poorly to vaccination, rendering them the most vulnerable population to preventable infectious diseases, such as influenza ${ }^{7}$ and viral hepatitis ${ }^{8,9}$.

Humoral immunity plays crucial roles in protecting hosts from microbial infections by generating antibodies ${ }^{10}$. During the immune response, naïve $\mathrm{B}$ cells encounter antigens and follicular $\mathrm{T}$ helper ( $\mathrm{Tfh}$ ) cells in the secondary lymph nodes (SLOs), which triggers their proliferation and differentiation into $\mathrm{CD} 27^{+}$memory $\mathrm{B}$ cells and antibody-secreting cells ${ }^{11}$. Under steady state conditions, human memory B cells, plasma cells, and antibody pools cross regulate each other in a balanced manner ${ }^{12}$. However, liver cirrhosis triggers an imbalance in these regulatory mechanisms. Depletion of the circulating $\mathrm{CD}_{2} 7^{+}$memory $\mathrm{B}$ cell pool and simultaneous hyperglobulinemia in cirrhotic patients have been

\section{(c) The Author(s) 2020}

(c) (i) Open Access This article is licensed under a Creative Commons Attribution 4.0 International License, which permits use, sharing, adaptation, distribution and reproduction cc) in any medium or format, as long as you give appropriate credit to the original author(s) and the source, provide a link to the Creative Commons license, and indicate if changes were made. The images or other third party material in this article are included in the article's Creative Commons license, unless indicated otherwise in a credit line to the material. If material is not included in the article's Creative Commons license and your intended use is not permitted by statutory regulation or exceeds the permitted use, you will need to obtain permission directly from the copyright holder. To view a copy of this license, visit http://creativecommons.org/licenses/by/4.0/. 
previously reported ${ }^{13-15}$. Interestingly, these phenomena have also been observed in various immune deficiency and autoimmune conditions ${ }^{16-21}$, suggesting possible common links underlying this imbalance.

Liver cirrhosis often causes increased spleen volume, and severe splenomegaly is treated through splenectomy ${ }^{22}$. The spleen is the largest SLO in the body and contains a large pool of immune cells, especially abundant $\mathrm{B}$ cells ${ }^{23}$. Although the spleen plays a key roles in immune defense against blood-borne infections ${ }^{24}$, the roles of the spleen in destroying immune cells or preventing harmful infections during liver cirrhosis are still under debate ${ }^{25}$. It is still unknown how CAID affects splenic immune cell compartments. Recently, we determined that an expanded hyperactive splenic $\mathrm{Tfh}$ cell population correlated with enhanced germinal center (GC) responses and hypergammaglobulinemia in cirrhotic patients ${ }^{26}$, showing the importance of investigating the regional immunity of the spleen in liver cirrhosis. Here, we comprehensively characterize the splenic B cell compartments from cirrhotic patients, investigate the underlying mechanisms of memory B cell depletion and functional impairment, and explore the consequences of these phenomena on the $\mathrm{B}$ cell repertoires and their potential clinical significance.

\section{Results}

The perturbations of peripheral and splenic B cell compartments in patients with liver cirrhosis

Although the peripheral $\mathrm{CD} 27^{+}$memory B cell pool is depleted in cirrhotic patients ${ }^{13-15,27}$, the splenic B cell compartments have not been well characterized. Here, spleen and peripheral blood samples were collected from cirrhotic patients (hepatitis B virus-associated cirrhosis (HBV-LC) and nonhepatitis B virus-associated cirrhosis (Non-HBV-LC)) and from healthy donors (Table 1) for a comprehensive splenic B cell study. Noncirrhotic patients with chronic hepatitis $\mathrm{B}(\mathrm{CHB})$ were also included in the peripheral $\mathrm{B}$ cell study for investigating the effect of $\mathrm{HBV}$ infection on B cells without cirrhotic conditions. The total splenic or peripheral B cell pools were investigated by a multicolor flow cytometric panel (Fig. 1a and Table 2, panel 1) that included CD45 and a group of B cell-related markers, such as CD19, CD21, CD27, CD10, CD38, IgD, $\operatorname{IgM}$, IgA, IgG, IgG1, IgG2, and IgG3. The flow cytometric data were analyzed by FlowJo software with UMAP to reduce the high-dimensional parameters to a $2 \mathrm{D} \mathrm{map}^{28}$. A total of eight subsets were identified based on UMAP clustering (Fig. 1b). Considering the similarities between the splenic and peripheral $\mathrm{B}$ cells, and considering the $\mathrm{B}$ cells subsets classifications by others ${ }^{29-32}$, these B cell subsets were named Trans\&GC B cells (transitional or germinal center (GC) B cells, CD $\left.38^{\text {hi }} \mathrm{CD} 10^{+}\right)$, PBs (plasmablasts, $\mathrm{CD} 38^{\mathrm{hi}} \mathrm{CD} 10^{-}$), MZB (marginal zone or marginal zone -like $\mathrm{B}$ cells, $\mathrm{CD} 27^{+} \operatorname{IgD}^{+} \operatorname{IgM}^{+}$), naïve $\mathrm{B}$ cells
$\left(\operatorname{IgD}{ }^{+} \operatorname{IgM}^{+} \mathrm{CD}_{27}^{-}\right)$, and $\mathrm{IgA} / \mathrm{G} 1 / \mathrm{G} 2 / \mathrm{G} 3$ memory B cells $\left(\mathrm{IgA}^{+}, \mathrm{IgG1}^{+}, \mathrm{IgG2}^{+}\right.$or $\mathrm{IgG3}^{+}$, and $\mathrm{IgD}^{-} \mathrm{IgM}^{-} \mathrm{CD} 27^{+/-}$). The Trans\&GC B cell and PB subsets differed from the other subsets due to their CD10 and CD38 expression, while the naive, $\mathrm{MZB}$ and $\mathrm{IgD}^{-}$memory $\mathrm{B}$ cells differed from the other subsets due to their IgD and CD27 expression patterns (Fig. 1c). Using this strategy, the frequencies of the total B cells and the B cell subsets from the spleen or periphery were analyzed, and compared between the patient groups and healthy controls. We found that the peripheral and splenic $\mathrm{CD} 19^{+} \mathrm{B}$ cell proportions were similar among the various groups (Fig. S1A). Importantly, both the peripheral blood and spleen from cirrhotic patients showed significant increases in Trans\&GC and naïve B cells, and showed a dramatic decreases in MZB and IgG1, IgG2, and IgG3 memory B cells (Fig. 1d-f). In accordance with our previous study ${ }^{26}$, plasmablasts were found to be elevated in the spleens of cirrhotic patients. IgA memory B cells were found to be significantly decreased in peripheral blood; however, their counterpart in the spleen seemed to have largely remained at a level comparable to that of the healthy subjects (Fig. 1e, f). Notably, while the CHB patients had broad, normal $B$ cell subsets similar to those of the healthy controls, their Trans\&GC B cells did show an increase similar to that of the cirrhotic patients (Fig. 1e), indicating a common B cell signature in patients with liver injury.

The reduction in splenic MZB cells is a prominent feature in the perturbed B cell compartment in LC. The frequency of MZB cells in the spleens of cirrhotic patients did not correlate with their disease severity indexes, such as the Child-Pugh score, white blood cell counts and platelet counts (Fig. S1B, C). However, the frequency of MZB cells in the spleens did correlate with several clinical parameters, including cholinesterase (CHE), creatine kinase (CK), hemoglobin, and red blood cell (RBC) counts (Fig. 1d). These data suggest that liver cirrhosis disturbs the B cell compartment not only in the periphery but also dramatically in the spleen.

\section{The persistent activation of splenic B cell compartments in cirrhotic patients}

The persistent activation of splenic Tfh cells may enhance $B$ cell maturation in cirrhotic patients ${ }^{26}$; therefore, we analyzed the activation-associated markers (CD95, FcRL4, CD86, and CD71) and migrationassociated markers (CD11c, CXCR3, and CCR6) on the $B$ cell subsets in LC patients (Table 2, panels 2 and 3). In these two panels, Trans\&GC B cells, PBs, naïve B cells, and MZB cells were gated according to their phenotypes, while $\mathrm{IgD}^{-}$memory $\mathrm{B}$ cells within $\mathrm{CD}^{-1-} 8^{+/} \mathrm{CD} 10^{-}$ mature B cells were divided into $\mathrm{CD} 27^{+}$classical memory $B$ cells (named cMBCs here) and $\mathrm{CD}_{2} 7^{-}$atypical memory B cells (named aMBCs here) ${ }^{33}$ (Figs. 1c and 
Table 1 Basic demographic information of the enrolled subjects.

\begin{tabular}{|c|c|c|c|c|}
\hline & $\mathrm{HC}$ & $\mathrm{CHB}$ & HBV-LC & Non-HBV-LC \\
\hline Subjects & 50 & 34 & 63 & 27 \\
\hline Spleen usage & 27 & 0 & 44 & $11^{\#}$ \\
\hline PBMC usage & ${ }_{27} \boldsymbol{\Delta}$ & 34 & ${ }_{24} \wedge$ & 16 \\
\hline Age (years) & $45.7(24-63)^{*}$ & $33.8(17-56)$ & $48.3(26-66)$ & $53.4(23-78)$ \\
\hline Gender (M/F) & $38 / 12$ & $26 / 8$ & $46 / 17$ & $7 / 20$ \\
\hline Platelet $\left(10^{9} / \mathrm{L}\right)$ & $176.3(5.5-440)$ & $201.7(69-377)$ & $79.2(9-404)$ & $101(25-233)$ \\
\hline Albumin (g/L) & $32.5(15.6-49)$ & $41.7(27-48)$ & $34.8(23-48)$ & $32.5(22-42)$ \\
\hline Tbil $(\mu \mathrm{M} / \mathrm{L})$ & $17.1(6.3-49.7)$ & $31.5(6.2-254.7)$ & $18.2(6.3-47.3)$ & $28.7(8.1-201.9)$ \\
\hline DBil $(\mu \mathrm{M} / \mathrm{L})$ & $8.5(1.9-39)$ & $20.5(2.1-200.7)$ & $8.2(2.4-38.1)$ & $16.3(3.4-165.3)$ \\
\hline $\operatorname{ALT}(U / L)$ & $51.7(9-476)$ & $154.4(8-969)$ & $25.9(7-136)$ & $32(11-86)$ \\
\hline AST (U/L) & $53.6(13.1-283)$ & $96.4(15-697)$ & $34.3(15-174)$ & 43.7 (14-109) \\
\hline $\operatorname{RBC}\left(10^{12} / \mathrm{L}\right)$ & $3.9(2.2-6.3)$ & $4.8(4-5.7)$ & $3.4(1.7-5.5)$ & $3.5(2.2-5.3)$ \\
\hline HGB $(g / L)$ & $112.6(62-182)$ & $144.7(108-172)$ & $95.2(29-164)$ & $101.7(53-154)$ \\
\hline CHE (1000 U/L) & $\begin{array}{l}5474.3 \\
(2150-11257)\end{array}$ & $\begin{array}{l}7847 \\
(3984-13373)\end{array}$ & $\begin{array}{l}4176.5 \\
(1696-8838)\end{array}$ & $\begin{array}{l}3960 \\
(1435-6906)\end{array}$ \\
\hline CK (U/L) & $453.8(41-1787)$ & NA & $66.1(19-153)$ & $90.4(23-844)$ \\
\hline HbeAg $(+/-)$ & NA & $27 / 7$ & $10 / 53$ & $0 / 27$ \\
\hline Child-Pugh class $(5 / 6 / 7 / 8)^{\$}$ & NA & NA & $8 / 11 / 16 / 9$ & $1 / 2 / 8 / 0$ \\
\hline
\end{tabular}

$M$ male, $F$ female, $T B i l$ total bilirubin, DBil direct bilirubin, $A L T$ alanine aminotransferase, $A S T$ aspartate aminotransferase, $R B C$ red blood cell, $H G B$ hemoglobin, $C H E$ cholinesterase, $C K$ creatine kinase, $H B e A g$ hepatitis Be antigen, NA not applicable.

"Clinical data of $\mathrm{HC}$ subjects were only from donors with spleen usage.

"The 11 nonHBV-LC patients included five PBC patients, two HCV-related patients, one alcoholic-related patient and three patients with cirrhosis of unknown reason.

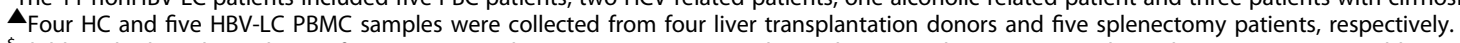

${ }^{\$}$ Child-Pugh class showed were from HBV-LC and Non-HBV-LC patients who underwent splenectomy to relieve the symptoms caused by portal hypertension and splenomegaly. The time of the clinical indexes collected for calculating Child-Pugh class were 1-2 weeks before the patients underwent splenectomy to avoid drugs affect.

2a). The expression of the activation-associated and migration-associated markers on the B cell subsets was then analyzed by gating the positive populations or by measuring the mean fluorescence intensity (MFI) (Fig. $2 \mathrm{~b}, \mathrm{c})$. Overall, regardless of whether the B cells were harvested from the periphery or the spleen, the B cell subsets from LC patients, to varying extents, expressed higher levels of CD95, FcRL4, CD86, CD71, CD11c, CXCR3, and CCR6 than those from HC subjects (Fig. S2A-D and Fig. 2d-g). In particular, CD95, a death receptor that is highly expressed on apoptotic cells and activated cells, was significantly upregulated on nearly all the splenic B cell subsets in LC patients (Fig. 2d). Similarly, the inhibitory receptor FcRL4 was also found to be significantly increased on the splenic MZB cells in LC patients (Fig. 2d). FcRL4 is known to be a receptor for commensal microbial antigens ${ }^{34}$. The increase in FcRL4 $^{+}$MZB cells may reflect the bacterial antigen accumulation or bacterial translocation in the spleens of LC patients. Interestingly, both CD86 and CD71 were found to be significantly increased on splenic Trans\&GC B cells from LC patients, while their patterns were different in the periphery (Fig. 2e and Fig. $\mathrm{S} 2 \mathrm{~B})$. As CD71 is also known to be a marker of pro-GC $\mathrm{B}$ cells ${ }^{35}$, this data suggests that splenic GC activity is enhanced during cirrhosis. Additionally, the upregulation of CD71 by peripheral B cells in CHB patients (Fig. S2B) was consistent with our previous findings ${ }^{36}$. $\mathrm{CD} 11 \mathrm{c}$, a marker for aMBCs that identifies an expanded $B$ cell subset in various chronic inflammatory conditions $^{37}$, was also increased in the splenic cMBCs, MZB cells, and aMBCs from nonHBV-LC patients (Fig. 2f). In addition, the splenic $\mathrm{cMBCs}$ and aMBCs from $\mathrm{LC}$ patients had higher levels of CXCR3 than those from $\mathrm{HC}$ subjects (Fig. 2f). Finally, compared with that in HC subjects, CCR6 expression was increased across multiple splenic B cell subsets in LC patients (Fig. 2g). Together, these results suggest a persistent activation of B cell compartments in cirrhotic patients, especially in the splenic B cell subsets. 


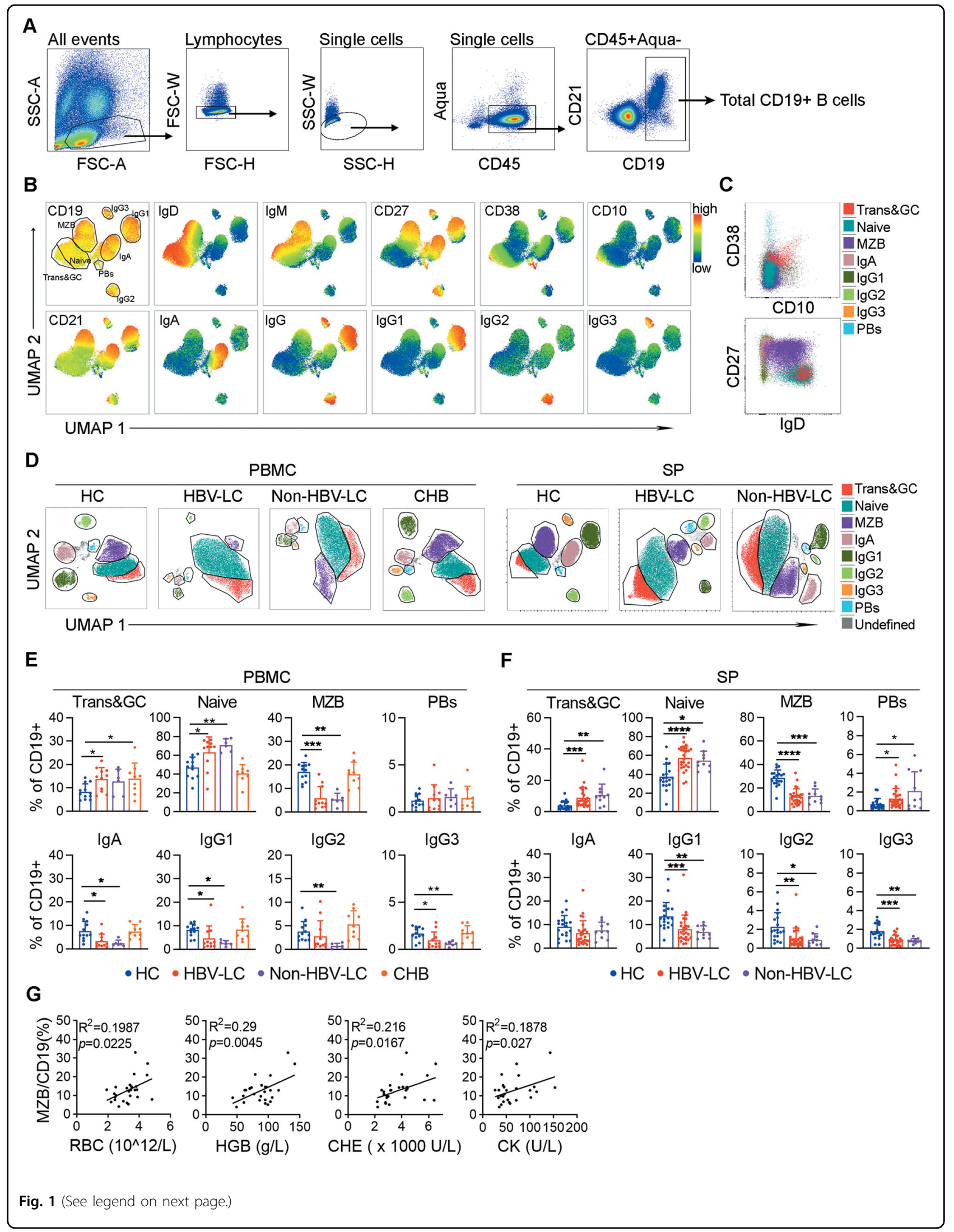


(see figure on previous page)

Fig. 1 Perturbations of splenic and peripheral B cell compartments in patients with liver cirrhosis. a Gating strategy for total B cells from PBMCs and spleens by flow cytometry. $\mathbf{b}$ Expression patterns of the indicated markers on total splenic B cells gated as in $\mathbf{a}$ using UMAP in FlowJo software. The plot was from the data of one representative healthy donor. Eight subsets were gated as indicated by the UMAP heat map. c Overlay of the 8 B cell subsets with a unique color for each subset based on CD38 and CD10 expression and/or CD27 and IgD expression. $\mathbf{d}$ Representative subset gating of the B cells from the PBMCs of healthy donors (HC), HBV-LC, nonHBV-LC, and CHB subjects (left) and from the spleens of HC, HBV-LC, and nonHBV-LC subjects (right) based on UMAP. e Frequencies of the B cell subsets from the PBMCs of HC $(n=12), \mathrm{HBV}-\mathrm{LC}(n=11)$, nonHBV-LC $(n=6)$, and CHB $(n=9)$ subjects. $\mathbf{f}$ Frequencies of the B cell subsets from the spleens of HC $(n=19)$, HBV-LC $(n=26)$ and nonHBV-LC $(n=10)$ subjects. ${ }^{*} P<0.05,{ }^{* *} P<0.01$, ${ }^{* *} P<0.001$, and ${ }^{* * *} P<0.0001$, as determined by Mann-Whitney U-test. $\mathbf{g}$ Correlation analysis of the splenic MZB frequencies and clinical markers, including red blood cells (RBCs), hemoglobin (HGB), cholinesterase (CHE) and creatine kinase (CK), in HBV-LC patients $(n=26)$. The Spearman rank correlation test was used, and $\mathrm{R}$ squared $\left(R^{2}\right)$ with $P$ values are shown.

Table 2 Antibody panels for flow cytometry.

\begin{tabular}{|c|c|c|c|}
\hline Marker & Clone & Fluorochrome & Panels \\
\hline CD45 & 2D1 & APC-H7 & Framework panel \\
\hline CD19 & $\mathrm{SJ} 25 \mathrm{Cl}$ & $\begin{array}{l}\text { BUV496, BUV395, } \\
\text { and BV711 }\end{array}$ & \\
\hline CD10 & HI10a & BV605 & \\
\hline CD21 & B-ly4 & PE-Cy7/PE-CF594 & \\
\hline CD27 & O323 & BV785 & \\
\hline CD38 & HIT2/HB7 & BV711/PE-Cy7 & \\
\hline $\lg D$ & IA6-2 & BV421 & \\
\hline $\lg M$ & $\begin{array}{l}\text { MHM-88/G20- } \\
127\end{array}$ & BV650/PE & \\
\hline Aqua & $\begin{array}{l}\text { (dead cell } \\
\text { exclusion) }\end{array}$ & BV510 & \\
\hline $\lg G$ & G18-145 & BUV395 & Panel 1 \\
\hline $\lg A$ & IS118E10 & PerCPVio700 & \\
\hline $\lg G 1$ & HP6001 & PE & \\
\hline $\lg G 2$ & HP6002 & AF647 & \\
\hline $\lg G 3$ & HP6050 & AF488 & \\
\hline CD95 & $\mathrm{DX} 2$ & PE & Panel 2 \\
\hline FCRL4 & 413D12 & PerCP-eFluor 710 & \\
\hline CD86 & IT2.2 & BV711/BV650 & \\
\hline CD11 & $\mathrm{S}-\mathrm{HCL}-3$ & APC & Panel 3 \\
\hline CXCR3 & $\mathrm{G} 025 \mathrm{H} 7$ & PcrCP-Cy5.5 & \\
\hline CCR6 & G034E3 & PE & \\
\hline CD71 & M-A712 & BV711 & \\
\hline
\end{tabular}

The enhanced GC response and decreased primary follicles in the spleens of cirrhotic patients

As stated above, LC patients have a significant increase of Trans\&GC B cells and loss of MZB cells and IgD $^{-}$ memory cells in their spleens. To further investigate these changes in situ, we conducted immunohistochemistry staining and examined serial splenic sections with anti-
CD20, anti-IgD, anti-Ki-67, and anti-CD1c, which were used to identify B cell follicles, splenic marginal zones, germinal centers and MZB cells ${ }^{30,38,39}$. We found that the spleens of cirrhotic patients generally showed enlarged GC-like secondary follicles (Fig. 3a). It is also obvious that $\operatorname{IgD}^{\text {hi }}$ naïve $\mathrm{B}$ cells $\left(\mathrm{CD} 1 \mathrm{c}^{-}\right)$aggregated at the mantle zone in the spleen, forming a distinct ringshaped layer surrounding the follicle center (Fig. 3a, b). Further staining of the B cell follicles with Ki-67 indicated increased proliferation in the pseudo-GC area in LC patients (Fig. 3a). While primary follicles showed comparable CD1c ${ }^{+}$cell enrichment (mainly MZB cells) in both $\mathrm{HC}$ and LC subjects, the enlarged secondary follicles in LC patients exhibited a relative thinner marginal zone than those in $\mathrm{HC}$ subjects (Fig. 3a, b). Moreover, LC patients obviously had a much lower number of primary B cell follicles, which was accompanied by increased secondary follicle counts (Fig. 3c, d). Interestingly, the primary follicles of LC patients were larger than those of $\mathrm{HC}$ subjects (Fig. 3e), likely reflecting a more active state. Those increased secondary structures are consistent with increased Trans\&GC $B$ cells, while the lack of primary structure accompanies MZB cell loss in LC spleens.

\section{Bulk RNA-seq revealed metabolic defects of the splenic B cells in LC patients}

To further explore the overall impact of liver cirrhosis on B cell subsets in LC patients, we performed bulk RNAseq on the sorted splenic naïve B cells, MZB cells and cMBCs from HBV-LC and HC subjects. We profiled the transcriptome of the $B$ cell subsets through principal component analysis (Fig. 4a). It is shown that the samples were clustered by B cell subsets rather than by subject groups. The transcriptomes of the MZB cells and cMBCs were more similar to each other but distinct from that of the naïve B cells. There were 41 upregulated and 65 downregulated genes observed in the naïve B cells, 30 upregulated and 87 downregulated genes observed in the MZB cells and 36 upregulated and 297 downregulated genes observed in the cMBCs in LC patients compared to those in $\mathrm{HC}$ subjects (Fig. 4b). 


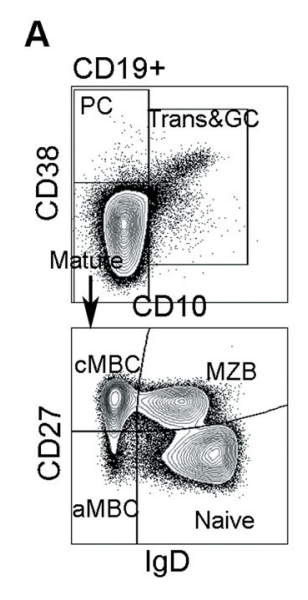

B $\frac{\mathrm{HC}}{\text { CD19(FMO)Trans\&GC Naive }}$

Cha

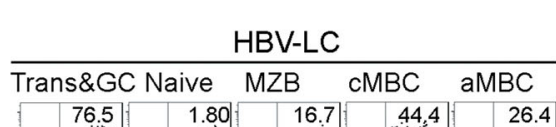

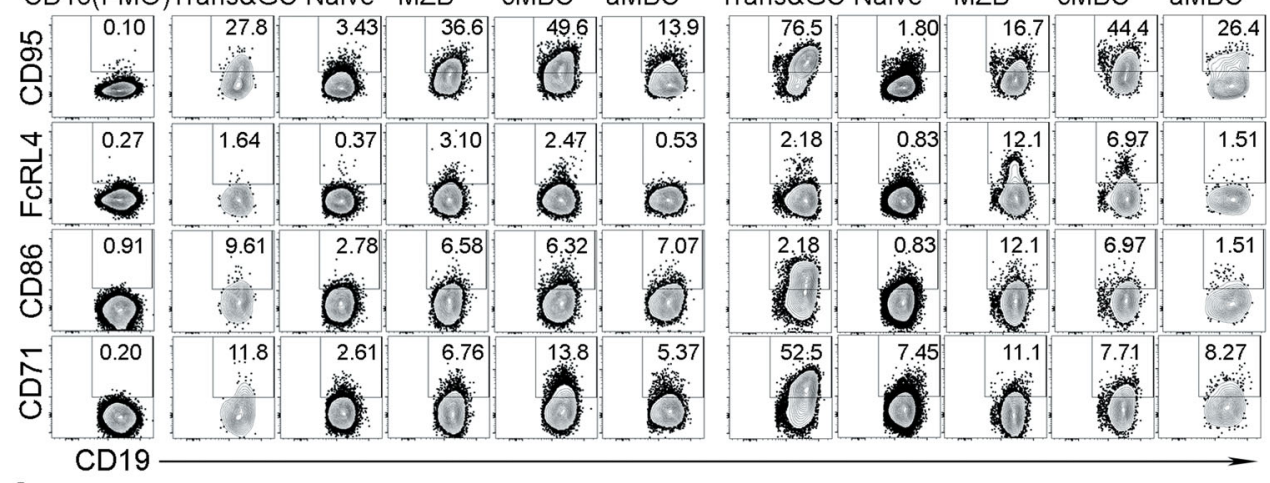

C

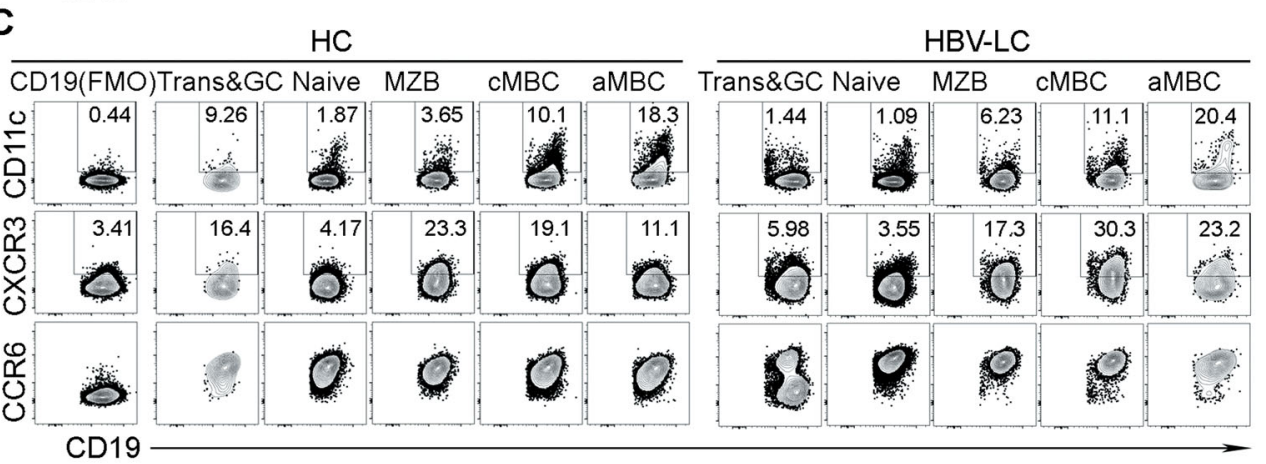

D
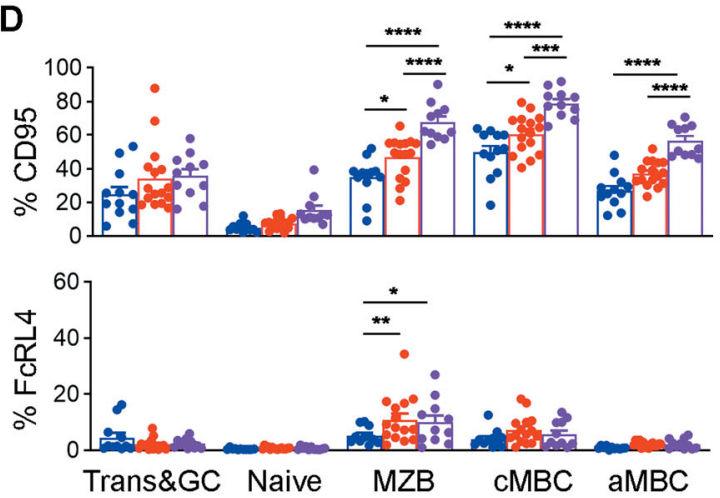

F
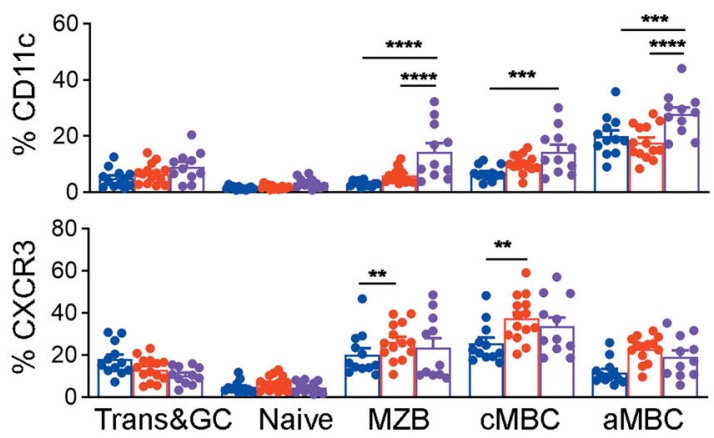

E
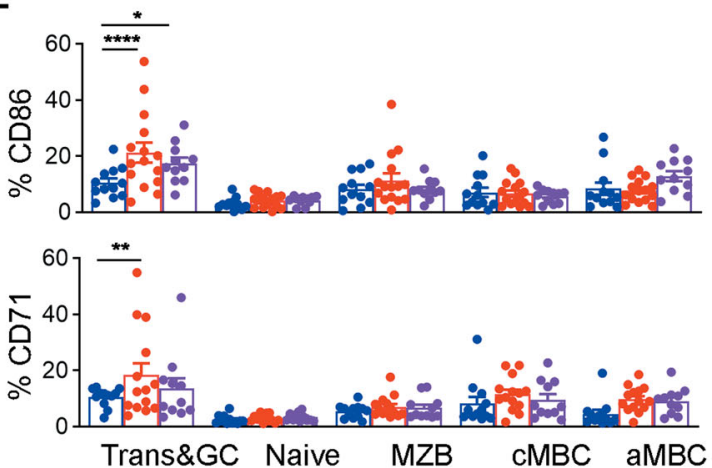

G

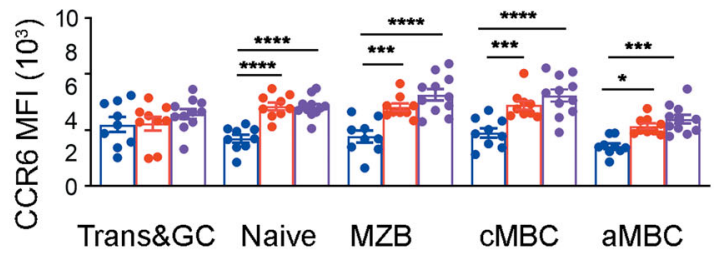

Fig. 2 (See legend on next page.) 
(see figure on previous page)

Fig. 2 Phenotypical analysis indicates the persistent activation of the spleen B cell compartments in LC patients. a Gating of Trans\&GC B cells, PBs, CMBCs, aMBCs, MZB cells, and naïve B cells on a FlowJo pseudocolor plot for subsequent phenotypic analysis. b Representative CD95, FCRL4, CD86, and CD71 expression profiles on the spleen B cell subsets from the spleens from one healthy donor and one HBV-LC donor. The positive populations were gated according to the Fluorescence Minus One (FMO) controls for each marker, and their percentages are indicated. c, d Percentages of the CD95 $5^{+}, \mathrm{FCRL4} 4^{+}, \mathrm{CD} 86^{+}$and $\mathrm{CD} 71^{+}$populations of Trans\&GC B cells, naïve B cells, MZB cells, CMBCs and aMBCs from the spleen of HC $(n=12)$, HBV-LC $(n=16)$, and nonHBV-LC $(n=11)$ subjects as indicated. e Representative CD11C, CXCR3, and CCR6 expression profiles of the spleen B cell subsets from the spleens of one healthy donor and one HBV-LC donor. $f$ Percentages of CD11 $C^{+}$and CXCR3 ${ }^{+}$populations of the B cell subsets of subjects as in $\mathbf{c}, \mathbf{d}$. $\mathbf{g}$ Mean fluorescence intensity (MFI) of CCR6 on the indicated B cell subsets from the spleens of HC $(n=9)$, HBV-LC (12) and nonHBV-LC $(n=11)$ subjects. Data are shown as the mean and sem. ${ }^{*} P<0.05,{ }^{* *} P<0.01,{ }^{* * *} P<0.001$, and ${ }^{* * * *} P<0.0001$, as determined by Mann-Whitney U-test.

Gene set enrichment analysis (GSEA) was used to identify the possible functional alterations in the B cell subsets from LC patients. Based on the hallmark gene sets in MSigDB, we confirmed that the mTORC1 signaling and oxidative phosphorylation pathways were significantly downregulated in all three B cell subsets from LC patients (Fig. 4c). The RNA-seq data also revealed a defect in glycolysis in splenic naïve B cells, MZBs and cMBCs of LC patients (Fig. S3A). Indeed, the expression levels of multiple genes in mTORC1 signaling, oxidative phosphorylation and glycolysis pathways were decreased (Fig. 4d and Fig. S3B). These results strongly suggest a broad metabolic defect of splenic B cells in LC patients. Furthermore, genes involved in the negative regulation of cell apoptosis and the positive regulation of cell proliferation were also downregulated in cMBCs and MZB cells in LC patients (Fig. S3C). Thus, we speculated that in cirrhosis, splenic B cells exhibit profound functional impairments in metabolism, antiapoptotic pathways, proliferation and/or differentiation.

\section{The functional impairment of B cell compartments from cirrhotic patients}

We further confirmed the defective metabolism of splenic B cells in LC patients. Glucose is dispensable for B cell activation, and oxidative phosphorylation is also fueled by other nutrients ${ }^{40}$. We found that the levels of glucose transporter GLUT1 on cMBC subset and the expression of CD98 (SLC3A2), a heavy chain of the heterodimeric amino acid transporter that was associated with proliferating cells ${ }^{41}$, by the MZB cell and aMBC subsets were significantly reduced in LC patients (Fig. 5a). In addition, CD36 (fatty-acid transporter) expression by all three B cell subsets was similar between LC patients and $\mathrm{HC}$ subjects (Fig. S4). However, we did observe significantly decreased uptake of fatty acids in naïve $\mathrm{B}$ cells, MZB cells, and cMBCs in LC patients (Fig. 5a). Mitochondrial dynamics play an important role in energy production of immune cells ${ }^{42}$. We also measured the splenic B cell mitochondrial mass to assess metabolic activity $^{43}$ using MitoTracker Green ${ }^{44}$ and observed a significant decrease in the cMBCs, MZB cells, and aMBCs of LC patients compared to that in $\mathrm{HC}$ subjects (Fig. 5a).
Moreover, we used Seahorse to analyze B cell oxygen consumption rate (OCR) and the extracellular acidification rate (ECAR), which measure mitochondrial energy production and lactate production by glycolysis, respectively, and we found that cultured B cells from cirrhotic patients have profound defects in oxygen consumption and glycolytic function in both untreated and stimulated conditions (Fig. 5b). In particular, there was significantly lower maximal respiratory potentials in the B cells from cirrhotic patients when stimulated by $\mathrm{CpG}$ (Fig. 5b). A $24 \mathrm{~h}$ culture also showed a decreased uptake of 2-(N-(7nitrobenz-2-oxa-1,3-diazol-4-yl)amino)-2-deoxyglucose (2-NBDG, a fluorescent glucose analog that is used to monitor glucose uptake in live cells) by splenic B cells from cirrhotic patients compared to those from healthy subjects (Fig. 5c). Together, these data indicated that liver cirrhosis likely caused profound impairment in utilizing amino acids and fatty acids for oxidative phosphorylation by the various splenic $B$ cell subsets.

We next examined whether the $B$ cells from cirrhotic patients exhibit functional impairments in mTORC1related signaling transduction, cytokine production and cell proliferation in response to stimulation. Indeed, we observed a significantly lower levels of pAKT and pS6 in HBV-LC splenic B cells stimulated by CpG or CpG/ CD40L (Fig. 5d), confirming a defect in mTORC1 activation. We also found that the cytokine production of the splenic B cell subsets was much lower in HBV-LC patients than in $\mathrm{HC}$ subjects (Fig. 5e). In addition, the splenic $B$ cells in HBV-LC patients displayed a much lower in vitro proliferation capacity after 4 days of culture than those in HC subjects (Fig. 5f). Thus, our data reveal that B cells from LC patients are hyperactive and functionally impaired and exhibit poor proliferative capacity, which are associated with their defective energy metabolism.

\section{Discussion}

Little is known regarding how liver cirrhosis affects splenic B cells. Here, we described the disturbed splenic B cell compartments and their hyperactivation phenotypes in LC patients. Our data also suggest that metabolic defects are the potential mechanisms for the splenic B cell 


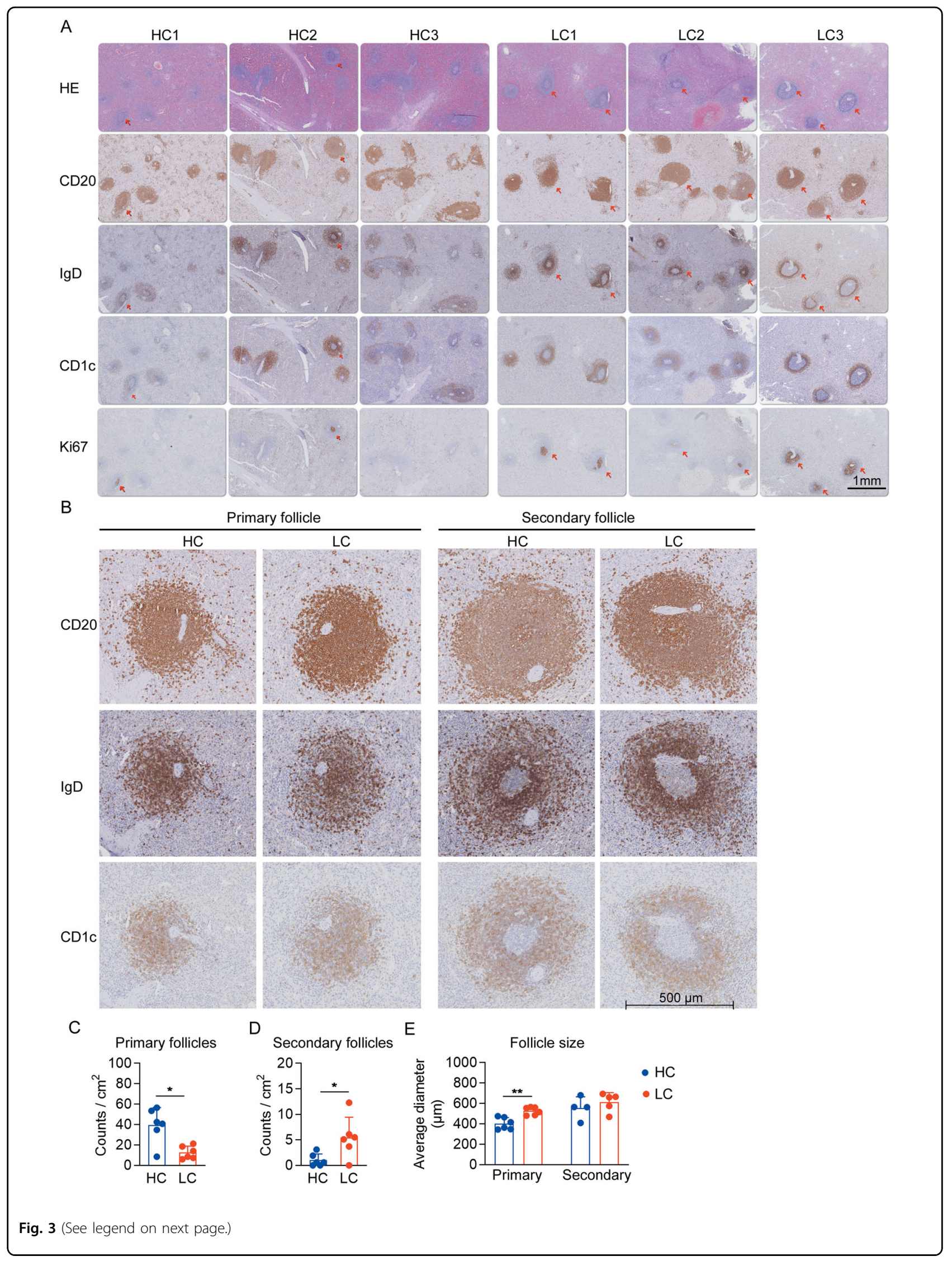


(see figure on previous page)

Fig. 3 Immunochemistry staining showing the enhanced GC responses and decreased MZB-enriched primary follicles in LC patients.

Formalin-fixed, paraffin-embedded splenic sections from healthy donors $(n=6)$ and LC patients $(n=6)$ were used for hematoxylin-eosin (HE) and immunohistochemical staining. a HE and CD20, lgD, CD1C and Ki-67 staining in serial/or adjacent splenic sections from three HC and three LC subjects. b Magnified representative primary and secondary follicles from HC and LC subjects stained with CD20, IgD, CD1c. c, d Average counts of the primary and secondary B cell follicles from HC $(n=6)$ and $L C(n=6)$ subjects. e Average diameter of the primary follicles from HC and LC subjects. Note that the secondary follicles could not be found in the sections from two HC and one $L C$ subjects. ${ }^{*} P<0.05$, and ${ }^{* *} P<0.01$, as determined by Mann-Whitney U-test.

functional impairment in LC patients. These defects in the $\mathrm{B}$ cell subsets may be linked to the diminished humoral immunity in cirrhotic patients.

The dysregulated B cell compartments are unique in cirrhotic patients and are different from the B cell compartments previously reported in other chronic inflammatory conditions, such as $\mathrm{HIV}, \mathrm{HCV}$, malaria, and autoimmunity ${ }^{16-21}$. Although the CD27 $7^{+}$memory B cells are significantly depleted, liver cirrhosis does not promote the expansion of $\mathrm{CD} 11 \mathrm{c}^{+}$Tbet $^{+}$aMBCs but significantly increases the naïve $B$ cell and Trans\&GC $B$ cell populations. In addition, $\mathrm{CD} 21$ expression in the $\mathrm{IgD}^{+/-} / \mathrm{CD} 27^{+}$ $B$ cell subsets was largely maintained in LC, which differs from other conditions ${ }^{16,20,21}$. The perturbations of splenic B cell compartments are not specific to HBV infection, since the $\mathrm{B}$ cell compartments in nonHBV cirrhosis also show similar perturbation as evident by the flowcytometry data. Together with other reports ${ }^{13}$, our data suggest a nonHBV-dependent mechanism underlying the dysregulation of B cell compartments during liver cirrhosis. Although it is possible that persistent HBV antigens may nonspecifically affect $\mathrm{B}$ cells ${ }^{45-47}$, we did not observe significant changes in the mature $\mathrm{B}$ cell compartments from the $\mathrm{CHB}$ patients enrolled in the current study. Future studies should examine whether HBV-associated antigens affect $B$ cell properties in larger patient cohorts with different clinical phases.

Persistent activation of spleen B cells are evident in LC patients, However, the mechanisms involved in memory $\mathrm{B}$ cell loss and functional impairment are not well understood. Here, we showed that defective metabolism is likely one of such missing links. Hyperinflammatory cues in LC patients may rewire the metabolic regulatory network. The bulk transcriptomic data reveal the broad defects in the cellular energy metabolic pathways in both the switched and nonswitched memory B cell subsets from LC patients; these data were subsequently confirmed by in vitro functional assays, which showed the impaired metabolic capacity and proliferation of the B cells. These phenomena are similar to the immune paralysis status reported in sepsis patients ${ }^{48}$, in which both glycolysis and oxidative metabolism are defective. In addition, the preferential loss of MZB cells is likely due to the increased activation and differentiation of $\mathrm{B}$ cells into plasma cells during LC. A previous study showed that $\operatorname{IgM}^{+} \mathrm{CD} 27^{+} \mathrm{B}$ cells have a higher capacity to migrate toward CXCL13 ${ }^{49}$, suggesting an ability of these cells to enter B cell follicles. Indeed, we observed a higher expression of CXCL13 in cirrhotic patients, and the increased splenic Tfh cells may facilitate the differentiation of plasma cells ${ }^{26}$. These data suggested that the increased engagement of MZB cells in GC reactions likely exhausts the splenic MZB cell pools during liver cirrhosis. Another possibility is increased activation-induced cell death, which is associated with the loss of $\mathrm{CD}_{2} 7^{+} \mathrm{B}$ cells ${ }^{50}$. Our transcriptomic analysis showed perturbation of the regulatory network related to $B$ cell death and survival in LC patients. Indeed, we observed the defective proliferative capacity of LC B cells. This did not contradict with the increased splenic ki-67 immunochemistry staining which concentrated in the pseudo-GC area and indicated mostly GC B cells. Thus, through the comprehensive analysis of the splenic $B$ cell subsets from LC patients, we proposed that defective and impaired metabolic capacities and heightened levels of differentiation and activation potentially exhaust the memory B cell pool and functionality.

SLOs are the sites where B cells respond to antigens. The spleen is the largest SLO and contains numerous B cells, particularly MZB cells ${ }^{23,24}$. Although the depletion of peripheral memory $B$ cells during liver cirrhosis has been reported ${ }^{3,13}$, little is known regarding the depletion of memory B cells in the SLOs. The splenic histology shows profound changes in B cell distributions along with increased naïve $B$ and decreased memory B cells in patients. It is interesting to speculate that the distinct naïve $B$ cell rings closely surrounding the GCs may affect the humoral immune response. More importantly, we identified a series of cirrhosisassociated pathways in splenic B cells for future characterization.

In summary, liver cirrhosis profoundly impacts B cell compartments, which may contribute to defective humoral immunity. A better understanding of this process may help to design intervention strategies to reinvigorate $\mathrm{B}$ cell functions.

\section{Materials and methods \\ Enrolled subjects and sample processing}

Cirrhotic spleen samples were collected from 44 hepatitis B virus-associated cirrhosis (HBV-LC) subjects and 


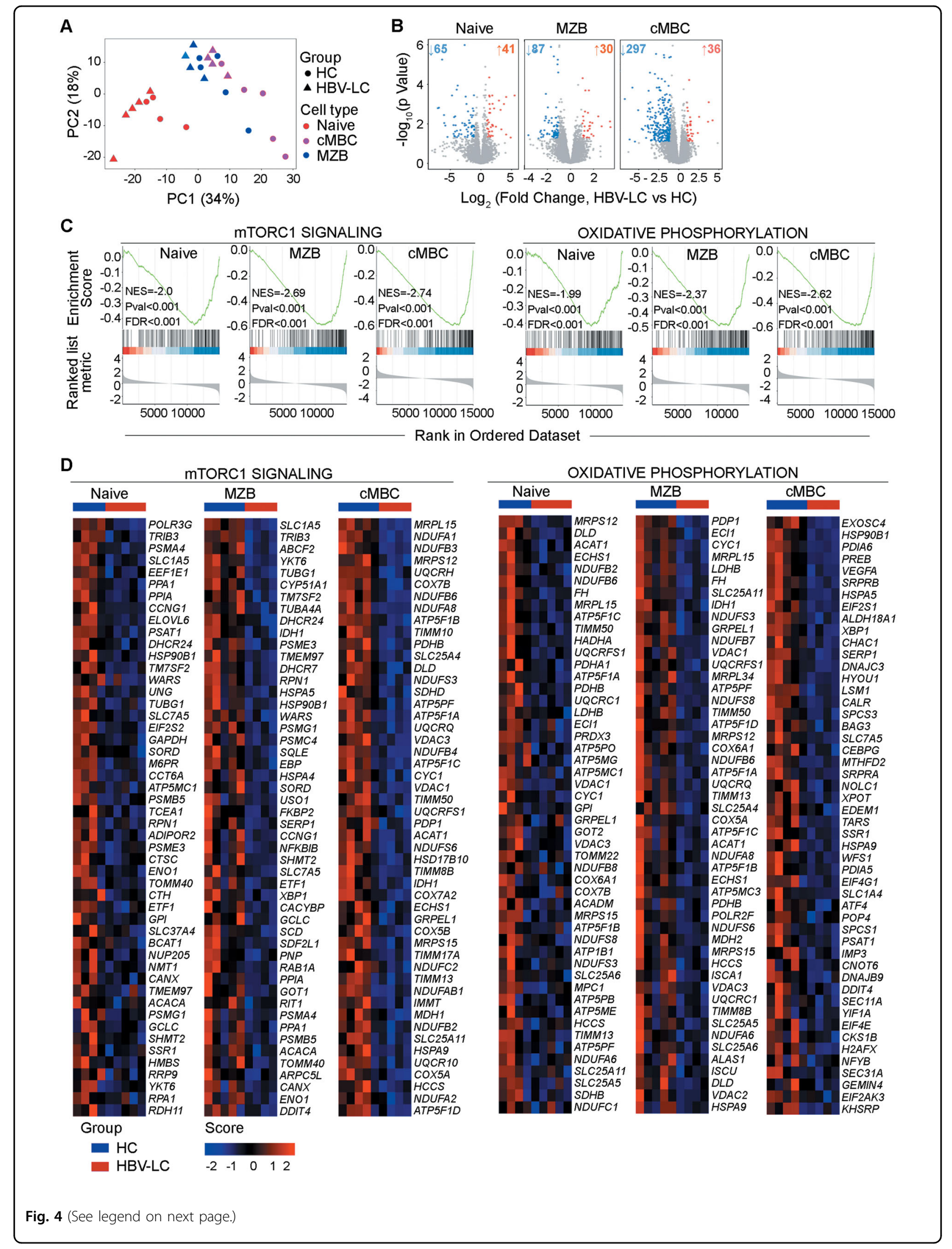


(see figure on previous page)

Fig. 4 Transcriptomic comparisons of the splenic B cell subsets in healthy donors versus LC patients. a PCA plot shows the transcriptomes of naïve $B$ cells, MZB cells, and CMBCs from HC and HBV-LC subjects. Each point represents one sample. $\mathbf{b}$ Volcano plots show the differentially expressed genes (DEGs) in the naïve B cells, MZB cells and CMBCs between HC and HBV-LC subjects. Each point represents one gene, and genes that had a $\log 2$ (foldchange) $<-1$ or $>1$ and $P$ value $<0.05$ as measured by Wald test were identified as downregulated or upregulated genes, respectively. The numbers of downregulated or upregulated genes are indicated. c GSEA plots show the enriched gene sets in the mTORC1 signaling, oxidative phosphorylation pathways, which were significantly downregulated in the naïve B cells, MZB cells, and CMBCs of HBV-LC subjects. The $X$-axis of the green curve is the rank of the genes that were ranked according to signal-to-noise values, and the $Y$-axis is the enrichment score (ES). The black bars indicate the rank of the genes in certain pathways. The genes on the right side of the peak indicate the leading edge subset genes that contribute the most to the ES. $\mathbf{d}$ Heatmaps show the genes (Top 50 arranged by signal to noise, see "Materials and methods" section) involved in the mTORC1 signaling and oxidative phosphorylation pathways that were downregulated in the naïve B, MZBs, and CMBCs from HBV-LC subjects compared with those from HC subjects.

11 non-hepatitis B virus-associated cirrhosis (nonHBVLC) subjects who underwent splenectomy to relieve the symptoms caused by portal hypertension and splenomegaly. Healthy spleen samples were collected from 27 liver transplantation donors as controls. Blood samples were taken from 24 HBV-LC, 16 nonHBV-LC, 34 chronic hepatitis $\mathrm{B}(\mathrm{CHB})$ patients and 27 age-matched healthy donors who had no evidence of infection, liver diseases, or autoimmune diseases. All patients and heathy donors were recruited from Shenzhen 3rd People's Hospital. Informed consent was obtained from all the participants. The protocol was approved by the ethics committee of Shenzhen 3rd People's Hospital (reference 2020-130). The basic demographic information of the enrolled subjects is listed in Table 1.

Spleen cells and PBMCs were isolated according to our previously published protocols ${ }^{26}$. For FACS staining, the cells were either used fresh or were cryopreserved. For bulk RNA sequencing, fresh cells were used. For the cell culture experiments, cryopreserved samples were used.

\section{Antibodies and flow cytometry}

The detailed information about the antibodies used to analyze the B cell subsets, phenotypes, and functions are listed in Supplementary Table 1. Fatty acid uptake was tested by BODIPY ${ }^{\mathrm{TM}}$ FL C16 (Invitrogen, Cat. D3821). To identify and exclude the dead cells, aqua fluorescent reactive dye (Invitrogen, Cat. L34957) was used according to the manufacturer's instructions. For surface marker staining, the cells were incubated with antibodies on ice for $30 \mathrm{~min}$ and then washed and fixed for further analysis. Intracellular staining was performed using the relevant antibodies in Perm/Wash Buffer (BD Biosciences) or the Foxp3/Transcription Factor Staining Buffer Set (eBioscience ${ }^{\mathrm{TM}}$, Cat. 005523). The samples were analyzed for B cell markers by FACS Frotessa and for metabolic markers by FACSCanto II flow cytometer (BD Biosciences), and the data were further analyzed by FlowJo software v.10 (TreeStar).

\section{Immunohistochemistry}

Hematoxylin-eosin (HE) and immunohistochemistry staining were performed using paraffin-embedded $4-\mu \mathrm{m}$ splenic tissue serial sections. The information about the primary mAbs is listed in Supplementary Table 1. The sections were first incubated with the optimal concentrations of the primary antibodies for $1 \mathrm{~h}$ at room temperature (RT). Then, the sections were incubated with the HRP-goat-rabbit/mouse antibodies (Cat. PV-6000, ZS-GB BIO) and were subsequently developed by DAB (Cat. ZLI-9018, ZS-GB BIO). All the slides were visualized using the NanoZoomer-XR Digital slide scanner C12000 under the $0.23 \mu \mathrm{m} /$ pixel mode, and the images were analyzed by NDP.view2 software. B cell follicles were determined by CD20 staining, with identification of primary follicles by no detection of germinal center formation, while the secondary follicles processed obvious germinal centers which were negative of IgD and positive of $\mathrm{Ki}-67^{30,38,39}$.

\section{Metabolic analysis}

MitoTracker Green (Invitrogen, Cat. M7541) was used to examine the mitochondrial mass. For MitoTracker Green staining and fatty acid uptake, splenic cells or magnetic bead-enriched B cells were resuspended in a 1/ 1000 dilution of Fixable Viability Dye eFluor ${ }^{\circledR} 450$ (Thermo Fisher). Then, the cells were incubated at room temperature for $15 \mathrm{~min}$, washed and resuspended with prewarmed, freshly made MitoTracker Green staining buffer $(50 \mu \mathrm{M})$ or BODIPY ${ }^{\mathrm{TM}}$ FL C16 staining buffer $(2 \mu \mathrm{M})$ at $37^{\circ} \mathrm{C}$ for an additional $30 \mathrm{~min}$. The cells were centrifuged, resuspended and stained with the other antibodies. The B cell oxygen consumption rate (OCR) and extracellular acidification rate (ECAR) were determined by a Seahorse XF platform according to the manufacturer's instructions (Agilent Technologies). 2-NBDG (Thermo Fisher, Cat. N13195) uptake was performed as described $^{51}$.

\section{mTOR1 signaling and cytokine production assay}

For assays examining pAKT and pS6 levels, enriched splenic B cells $\left(1 \times 10^{6}\right.$ cells $\left./ \mathrm{mL}\right)$ were cultured unstimulated or stimulated with CpG ODN2006 $(1 \mu \mathrm{M})$, CpG $(1 \mu \mathrm{M})$ plus rapamycin (Rapa, $10 \mathrm{nM})$ or CpG $(1 \mu \mathrm{M})$ plus CD40L $(500 \mathrm{ng} / \mathrm{mL})$ for $10 \mathrm{~h}$, then pAKT (Thr308) and 


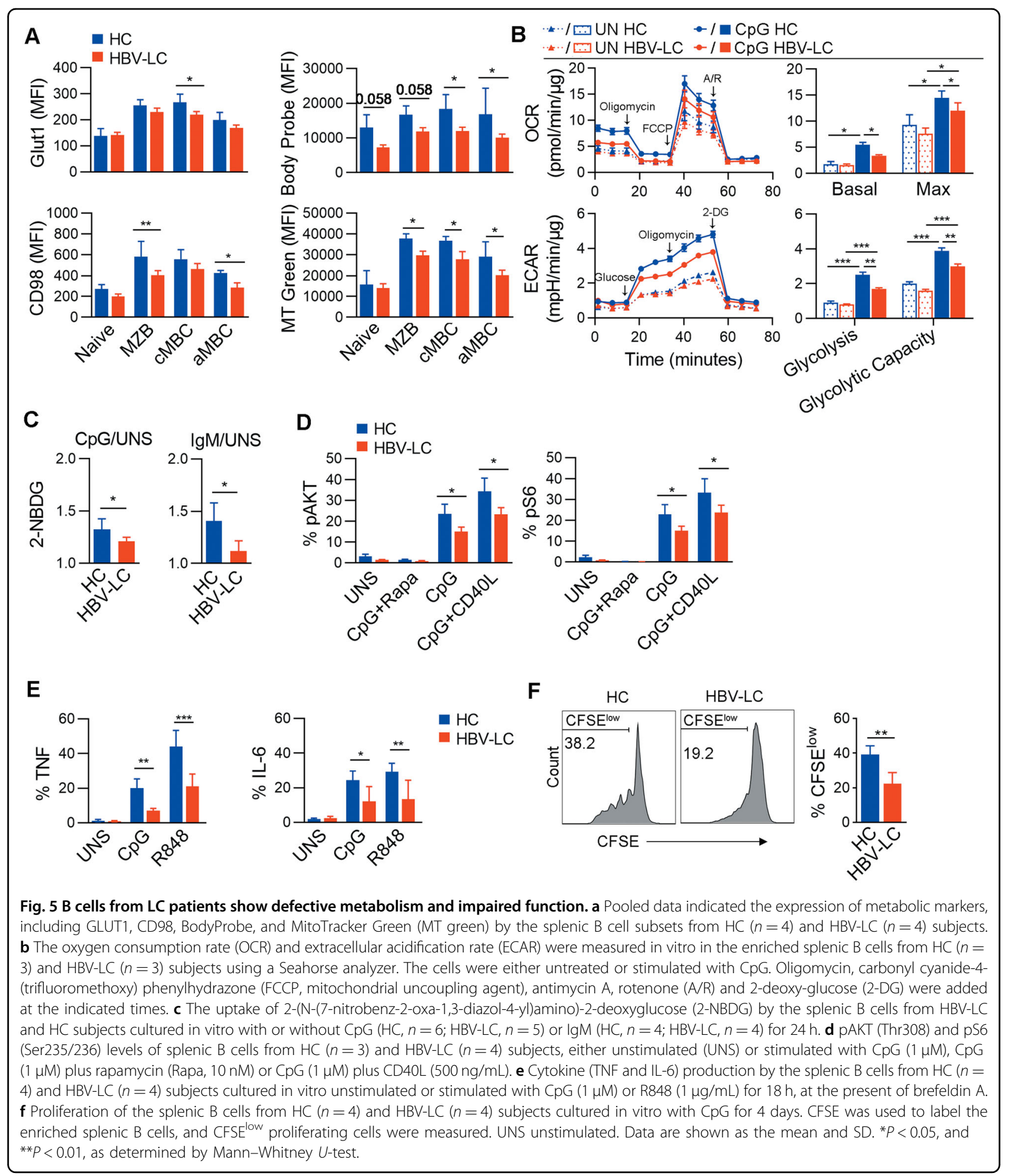

pS6 (Ser235/236) were analyzed by intracellular staining with PE anti-pAKT and AF488 anti-pS6. For cytokine production assay, enriched splenic $B$ cells were stimulated by CpG $(1 \mu \mathrm{M})$ or medium with BFA $(1 \mu \mathrm{g} / \mathrm{mL})$ for $18 \mathrm{~h}$ in 96-well U-bottom plates (Costar, Cat. 3799). Then, the cells were collected for surface marker staining, followed by intracellular staining for TNF and IL-6, and analyzed by flow cytometry. 
In vitro $B$ cell proliferation and differentiation assay

The enriched splenic B cells were labeled with CFSE and cultured in 96-well U-bottom plates with or without CpG $(1 \mu \mathrm{M})$ at a concentration of $1 \times 10^{6}$ cells $/ \mathrm{mL}$ for 4 days. Then, the proliferating cells $\left(\mathrm{CFSE}^{\text {low }}\right)$ were analyzed by flow cytometry.

\section{Sample processing for bulk RNA-seq}

For bulk RNA-seq, freshly isolated B cells were enriched using a B Cell Isolation Kit II (Miltenyi Biotec, Cat. 130091-151). Then, the enriched B cells were stained with anti-CD19 PerCP-Cy5, anti-CD10 PE, anti-CD27 PE-Cy7, anti-IgD PE-CF594, and anti-IgM APC for $30 \mathrm{~min}$, washed and resuspended at a concentration of $10^{7}$ cells/ $\mathrm{mL}$. A FACS Aria II cell sorter was used to sort $\mathrm{CD} 19^{+} \mathrm{CD} 10^{-} \mathrm{IgD}^{+} \mathrm{IgM}^{+} \mathrm{CD} 27^{+} \quad$ MZB cells, $\mathrm{CD}_{19}{ }^{+} \mathrm{CD} 10^{-} \mathrm{IgD}^{+} \mathrm{IgM}^{+} \mathrm{CD} 27^{-}$naïve $\mathrm{B}$ cells, and $\mathrm{CD}_{19}{ }^{+} \mathrm{CD} 10^{-} \mathrm{IgD}^{-} \mathrm{IgM}^{-} \mathrm{CD} 27^{+}$cMBCs. At least $1 \times 10^{6}$ cells were collected for each sample. The collected B cell subsets were centrifuged after a purity check on the sorter, and the subsets were suspended in TRIzol and stored at $-80{ }^{\circ} \mathrm{C}$ until RNA extraction. A total of 27 samples from five $\mathrm{HC}$ subjects and five HBV-LC patients were ultimately collected for bulk RNA-seq.

\section{Bulk RNA-seq mapping and GSEA}

The samples were sequenced on an Illumina HiSeq X Ten platform. Paired-end libraries were constructed with an insert size of $250-300 \mathrm{bp}$. The length of each read was $150 \mathrm{bp}$. The clean reads were mapped to the human genome (hg38) by applying HISAT2 ${ }^{52}$ with the default parameter. Then, we calculated the gene count using feature counts of SUBREAD ${ }^{53}$ with default parameters. The genes with fewer than ten gene counts were discarded. Then, the gene abundances were compared using the $\mathrm{R}$ package of DESeq $2^{54}$. PCA was also performed by DESeq2 with rlog normalized gene abundance. Differentially expressed genes (DEGs) were calculated by Wald test, by which genes that had a $\log _{2}$ (foldchange) of $<-1$ or $>1$ ( $p$ value $<0.05$ ) were identified as downregulated or upregulated genes, respectively. GO analysis was performed in DAVID (https://david.ncifcrf.gov/) using the differentially expressed genes. The GSEA was performed using the $\mathrm{R}$ package of clusterProfiler (Version 3.12.0) ${ }^{55}$, in which the hallmark gene sets in $\mathrm{MSigDB}^{56}$ were used for annotation. Genes for GSEA were ranked by signal to noise ratio, with the formula $\mu_{\mathrm{A}}-\mu_{\mathrm{B}} / \sigma_{\mathrm{A}}-\sigma_{\mathrm{B}}$, where $\mu$ is the mean and $\sigma$ is the standard deviation; $\sigma$ has a minimum value of 0.2 .

\section{Statistics}

All the statistical analysis was performed using GraphPad Prism Version 8.0.2. The data are presented as the mean values with SDs or SEMs. Multiple comparisons among the different groups were first performed using the nonparametric Kruskal-Wallis $H$-test. Comparisons between various groups were made using the Mann-Whitney $U$-test, whereas comparisons between the same individual were made using Wilcoxon's matched-pairs test. Correlations between two variables were analyzed using the Spearman rank correlation test. $P<0.05$ on two sides was considered to be significant for all the analyses.

\section{Acknowledgements}

This work was supported in part by grants from the National Science and Technology Major Project of the Infectious Diseases (2018ZX10301404 to Z.Z. and $2017 Z X 10202202$ to J.S.), the National Natural Science Foundation of China (81974259 to S.Z., 81871668 to J.S., and 81672037 to J.Z.), the Science and

Technology Innovation Committee of Shenzhen Municipality (JCYJ20170412151722110 to Z.Z. and JCYJ20170412151650600 to J.Z.), Guangzhou Science and Technology Plan Project (201804020001 to J.S.), Local Innovative and Research Teams Project of Guangdong Pearl River Talents Program (2017BT01S131 to J.S.).

\section{Author details}

${ }^{1}$ Institute for Hepatology, National Clinical Research Center for Infectious Disease, Shenzhen Third People's Hospital, 518112 Shenzhen, Guangdong, China. ${ }^{2}$ The Second Affiliated Hospital, School of Medicine, South University of Science and Technology, 518100 Shenzhen, China. ${ }^{3}$ Institute of Biophysics, Chinese Academy of Sciences, 100101 Beijing, China. ${ }^{4}$ Department of Infectious Diseases, Nanfang Hospital, Southern Medical University, 510515 Guangzhou, China. 5 State Key Laboratory of Organ Failure Research,

Guangdong Provincial Key Laboratory of Viral Hepatitis Research, Nanfang Hospital, Southern Medical University, 510515 Guangzhou, China. ${ }^{6}$ Research Center for Clinical \& Translational Medicine, Fifth Medical Center for General Hospital of PLA, 100039 Beijing, China. ${ }^{7}$ Department for Liver Transplantation, Fifth Medical Center for General Hospital of PLA, 100039 Beijing, China.

${ }^{8}$ Department for Liver Transplantation, Shenzhen Third People's Hospital, 518112 Shenzhen, Guangdong, China. ${ }^{9}$ Guangdong Key Lab of Emerging Infectious Diseases, Shenzhen Third People's Hospital, 518112 Shenzhen, China. ${ }^{10}$ Shanghai Public Health Clinical Center, Fudan University, 201508 Shanghai, China. ${ }^{11}$ Institute of Hepatology, Shenzhen 3rd People's Hospital, 5181112 Shenzhen, Guangdong Province, China

\section{Author contributions}

S.Z., J.S., and Z.Z. designed the study; M.H., and X.L. did the flow cytometry, immunohistochemistry and functional experiments; M.H., X.L., and B.H. analyzed the flow cytometry data; J.Z., X.H., and M.Q. did the RNA sequencing experiments; H.Y., and Y.L. analyzed bulk RNA-seq; J.P., and X.Z. checked case history; X.Z., Z.L., Z.L., Y.C., and L.L., provided human samples; S.Z. and Z.Z. wrote the manuscript.

\section{Conflict of interest}

The authors declare that they have no conflict of interest.

\section{Publisher's note}

Springer Nature remains neutral with regard to jurisdictional claims in published maps and institutional affiliations.

Supplementary Information accompanies this paper at (https://doi.org/ 10.1038/s41419-020-03060-1).

Received: 13 August 2020 Revised: 23 September 2020 Accepted: 24 September 2020

Published online: 24 October 2020

\section{References}

1. Strnad, P., Tacke, F., Koch, A. \& Trautwein, C. Liver-guardian, modifier and target of sepsis. Nat. Rev. Gastroenterol. Hepatol. 14, 55-66 (2017).

2. Tsochatzis, E. A., Bosch, J. \& Burroughs, A. K. Liver cirrhosis. Lancet 383, 1749-1761 (2014). 
3. Albillos, A., Lario, M. \& Alvarez-Mon, M. Cirrhosis-associated immune dysfunction: distinctive features and clinical relevance. J. Hepatol. 61, 1385-1396 (2014).

4. Acharya, C. \& Bajaj, J. S. Altered microbiome in patients with cirrhosis and complications. Clin. Gastroenterol. Hepatol. 17, 307-321 (2019).

5. Lange, C. M. \& Moreau, R. Immunodysfunction in acute-on-chronic liver failure Visc. Med. 34, 276-282 (2018).

6. Invine, K. M., Ratnasekera, I., Powell, E. E. \& Hume, D. A. Causes and consequences of innate immune dysfunction in cirrhosis. Front. Immunol. 10, 293 (2019).

7. Leise, M. D. \& Talwalkar, J. A. Immunizations in chronic liver disease: what should be done and what is the evidence. Curr. Gastroenterol. Rep. 15, 300 (2013).

8. Aggeletopoulou, I., Davoulou, P., Konstantakis, C., Thomopoulos, K. \& Triantos, C. Response to hepatitis B vaccination in patients with liver cirrhosis. Rev. Med. Virol. https://doi.org/10.1002/rmv.1942 (2017).

9. Zhang, S. et al. Investigation of underlying comorbidities as risk factors for symptomatic human hepatitis E virus infection. Aliment. Pharmacol. Ther. 45, 701-713 (2017)

10. Lu, L. L., Suscovich, T. J., Fortune, S. M. \& Alter, G. Beyond binding: antibody effector functions in infectious diseases. Nat. Rev. Immunol. 18, 46-61 (2018)

11. Crotty, S. T. Follicular helper cell biology: a decade of discovery and diseases. Immunity 50, 1132-1148 (2019).

12. Inoue, T., Moran, I., Shinnakasu, R., Phan, T. G. \& Kurosaki, T. Generation of memory B cells and their reactivation. Immunol. Rev. 283, 138-149 (2018).

13. Doi, H. et al. Dysfunctional B-cell activation in cirrhosis resulting from hepatitis C infection associated with disappearance of CD27-positive B-cell population. Hepatology 55, 709-719 (2012).

14. Doi, H., Tanoue, S. \& Kaplan, D. E. Peripheral CD27-CD21- B-cells represent an exhausted lymphocyte population in hepatitis C cirrhosis. Clin. Immunol. 150, 184-191 (2014).

15. Chang, L. Y., Li, Y. \& Kaplan, D. E. Endotoxemia contributes to CD27+ memory B-cell apoptosis via enhanced sensitivity to Fas ligation in patients with Cirrhosis. Sci. Rep. 6, 36862 (2016).

16. Thorarinsdottir, K., Camponeschi, A., Gjertsson, I. \& Martensson, I. L. CD21 -/low B cells: a snapshot of a unique B cell subset in health and disease. Scand. J. Immunol. 82, 254-261 (2015).

17. Portugal, S., Obeng-Adjei, N., Moir, S., Crompton, P. D. \& Pierce, S. K. Atypical memory $B$ cells in human chronic infectious diseases: an interim report. Cell Immunol. 321, 18-25 (2017).

18. Ubillos, I. et al. Chronic exposure to malaria is associated with inhibitory and activation markers on atypical memory $B$ cells and marginal zone-like B cells. Front. Immunol. 8, 966 (2017)

19. Carrillo, J. et al. Memory B cell dysregulation in HIV-1-infected individuals. AIDS 32, 149-160 (2018)

20. Liechti, T. et al. Widespread B cell perturbations in HIV-1 infection afflict naive and marginal zone B cells. J. Exp. Med. 216, 2071-2090 (2019).

21. Borhis, G. \& Richard, Y. Subversion of the B-cell compartment during parasitic, bacterial, and viral infections. BMC Immunol. 16, 15 (2015).

22. Boyer, T. D. \& Habib, S. Big spleens and hypersplenism: fix it or forget it? Liver Int. 35, 1492-1498 (2015).

23. Mebius, R. E. \& Kraal, G. Structure and function of the spleen. Nat. Rev. Immunol. 5, 606-616 (2005).

24. Lewis, S. M., Williams, A. \& Eisenbarth, S. C. Structure and function of the immune system in the spleen. Sci. Immunol. https://doi.org/10.1126/ sciimmunol.aau6085 (2019)

25. Li, L. et al. The spleen in liver cirrhosis: revisiting an old enemy with novel targets. J. Transl. Med. 15, 111 (2017).

26. Zhao, J. et al. Hyperactive follicular helper $\mathrm{T}$ cells contribute to dysregulated humoral immunity in patients with liver cirrhosis. Front. Immunol. 10, 1915 (2019).

27. Santer, D. M. et al. Enhanced activation of memory, but not naive, B cells in chronic hepatitis $C$ virus-infected patients with cryoglobulinemia and advanced liver fibrosis. PLOS ONE 8, e68308 (2013).

28. Mclnnes, L., Healy, J., Saul, N. \& Großberger, L. UMAP: Uniform Manifold Approximation and Projection. J. Open Source Softw. 3, 861, https://doi.org/ 10.21105/joss.00861 (2018).

29. Finak, G. et al. Standardizing flow cytometry immunophenotyping analysis from the human immunophenotyping consortium. Sci. Rep. https://doi.org/ 10.1038/srep20686 (2016).
30. Weller, S. et al. Human blood lgM "memory" B cells are circulating splenic marginal zone $B$ cells harboring a prediversified immunoglobulin repertoire Blood 104, 3647-3654 (2004).

31. Ubillos, I. et al. Chronic exposure to malaria is associated with inhibitory and activation markers on atypical memory B cells and marginal zone-like B cells. Front. Immunol. https://doi.org/10.3389/fimmu.2017.00966 (2017).

32. Clavarino, G. et al. Novel strategy for phenotypic characterization of human B lymphocytes from precursors to effector cells by flow cytometry. PLoS ONE 11, e0162209 (2016).

33. Weisel, F., Shlomchik, M. \& Memory, B. Cells of mice and humans. Annu Rev. Immunol. 35, 255-284 (2017).

34. Liu, Y. et al. Antibodies encoded by FCRL4-bearing memory B cells preferentially recognize commensal microbial antigens. J. Immunol. $\mathbf{2 0 0}$ 3962-3969 (2018)

35. Kolar, G. R., Mehta, D., Pelayo, R. \& Capra, J. D. A novel human B cell subpopulation representing the initial germinal center population to express AID. Blood 109, 2545-2552 (2007).

36. $\mathrm{Xu}, \mathrm{X}$. et al. Reversal of B-cell hyperactivation and functional impairment is associated with $\mathrm{HBsAg}$ seroconversion in chronic hepatitis B patients. Cell. Mol. Immunol. 12, 309-316 (2015).

37. Wang, S. et al. IL-21 drives expansion and plasma cell differentiation of autoreactive CD11c(hi)T-bet(+) B cells in SLE. Nat. Commun. 9, 1758 (2018).

38. Chadburn, A. The spleen: anatomy and anatomical function. Semin. Hematol. 37, 13-21 (2000).

39. Daridon, $\mathrm{C}$. et al. Splenic proliferative lymphoid nodules distinct from germinal centers are sites of autoantigen stimulation in immune thrombocytopenia. Blood 120, 5021-5031 (2012).

40. Waters, L. R., Ahsan, F. M., Wolf, D. M., Shirihai, O. \& Teitell, M. A. Initial B cell activation induces metabolic reprogramming and mitochondrial remodeling. iscience 5, 99-109 (2018).

41. Cantor, J. M. \& Ginsberg, M. H. CD98 at the crossroads of adaptive immunity and cancer. J. Cell Sci. 125, 1373-1382 (2012).

42. Xie, J.-H., Li, Y.-Y. \& Jin, J. The essential functions of mitochondrial dynamics in immune cells. Cell. Mol. Immunol. 17, 712-721 (2020).

43. Mitra, K., Wunder, C., Roysam, B., Lin, G. \& Lippincott-Schwartz, J. A hyperfused mitochondrial state achieved at G1-S regulates cyclin E buildup and entry into S phase. Proc. Natl Acad. Sci. USA 106, 11960-11965 (2009).

44. Agnello, M., Morici, G. \& Rinaldi, A. M. A method for measuring mitochondrial mass and activity. Cytotechnology 56, 145-149 (2008).

45. Burton, A. R. et al. Circulating and intrahepatic antiviral B cells are defective in hepatitis B. J. Clin. Investig. 128, 4588-4603 (2018).

46. Poonia, B., Ayithan, N., Nandi, M., Masur, H. \& Kottilil, S. HBV induces inhibitory FCRL receptor on B cells and dysregulates B cell-T follicular helper cell axis. Sci. Rep. 8, 15296 (2018).

47. Salimzadeh, L. et al. PD-1 blockade partially recovers dysfunctional virusspecific B cells in chronic hepatitis B infection. J. Clin. Investig. 128, 4573-4587 (2018).

48. Cheng, S. C. et al. Broad defects in the energy metabolism of leukocytes underlie immunoparalysis in sepsis. Nat. Immunol. 17, 406-413 (2016).

49. Seifert, $M$. et al. Functional capacities of human IgM memory B cells in early inflammatory responses and secondary germinal center reactions. Proc. Natl Acad. Sci. USA 112, E546-E555 (2015).

50. Shankar-Hari, M. et al. Activation-associated accelerated apoptosis of memory B cells in critically III patients with sepsis. Crit. Care Med. $\mathbf{4 5}$, 875-882 (2017).

51. Gemta, L. F. et al. Impaired enolase 1 glycolytic activity restrains effector functions of tumor-infiltrating CD8(+) T cells. Sci. Immunol. https://doi.org/ 10.1126/sciimmunol.aap9520 (2019).

52. Kim, D., Paggi, J. M., Park, C., Bennett, C. \& Salzberg, S. L. Graph-based genome alignment and genotyping with HISAT2 and HISAT-genotype. Nat. Biotechnol. 37, 907-915 (2019).

53. Liao, Y., Smyth, G. K. \& Shi, W. The subread aligner: fast, accurate and scalable read mapping by seed-and-vote. Nucleic Acids Res. 41, e108 (2013).

54. Love, M. I., Huber, W. \& Anders, S. Moderated estimation of fold change and dispersion for RNA-seq data with DESeq2. Genome Biol. 15, 550 (2014).

55. Yu, G., Wang, L. G., Han, Y. \& He, Q. Y. clusterProfiler: an R package for comparing biological themes among gene clusters. Omics 16, 284-287 (2012).

56. Liberzon, A. et al. The Molecular Signatures Database (MSigDB) hallmark gene set collection. Cell Syst. 1, 417-425 (2015). 